\title{
Negative Symptoms of Schizophrenia: Not All is Negative!
}

\author{
Jagadisha Thirthalli
}

Published online: 21 July 2021

(C) The Author(s), under exclusive licence to Springer Nature India Private Limited 2021

That schizophrenia is one of the most disabling conditions is an oft-quoted, cliched sentence. Despite advances in therapeutics, schizophrenia continues to disable a substantial number of people in the prime of their youth across the Globe. As with other disabling health conditions, disability in schizophrenia is due to a complex interplay between the health condition and contextual factors. Among the factors related to the condition per se, negative symptoms are of considerable importance. During the onset of the illness and during relapses of psychotic episodes, positive symptoms cause major disruption in the lives of persons with schizophrenia. They frequently lead to hospitalization, break in education or occupation and break in personal relationships. However, interventions to address positive symptoms - largely antipsychotic medications and clinical care - are reasonably effective.

However, considerable proportion of individuals with schizophrenia experience long-lasting negative symptoms [1]. Affective flattening, alogia, anhedonia, asociality, avolition, etc., frequently last throughout the course of the illness, particularly through the medium and long term, and contribute significantly to disability. Assessment of the severity of negative symptoms is, to

J. Thirthalli $(\bowtie)$

Psychiatric Rehabilitation Services (PRS), Department of Psychiatry, National Institute of Mental Health and Neurosciences (NIMHANS), Bengaluru 560029, India e-mail: jagatth@yahoo.com some extent, based on assessment of the individual's social functioning, and this may partly explain the association between negative symptoms and social functioning. For instance, in the Positive and Negative Syndrome Scale (PANSS) [2], 'emotional withdrawal' (N2) is assessed based on "reports of functioning from primary care workers or family" in addition to observation during the interview; "passive apathetic withdrawal' (N4) is assessed based on "reports on social behavior from primary care workers or family". These very elements of information are used to assess social functioning as well. Thus, high association between the two is unsurprising. Notwithstanding such methodological considerations, one cannot discount the fact that negative symptoms add to the burden of disability associated with schizophrenia.

Pessimism about negative symptoms The mention of negative symptoms evokes nihilistic response from experienced mental health professionals. There are at least two obvious reasons for this deeply entrenched opinion about negative symptoms-neurobiology underlying these symptoms and dearth of evidencebased treatment options.

Neurobiology of negative symptoms is a lot less well understood in comparison to that of positive symptoms. A substantial part of this lack of knowledge is owing to the heterogeneity in the way negative symptoms are conceptualized. Nevertheless, there is consistent evidence that deficient dopamine transmission is associated with negative symptoms [3]. 
Antipsychotic medications, the mainstay of treatment of schizophrenia happen to be dopamine blockers and, as such, are not expected help in the treatment of negative symptoms. Atypical antipsychotics, with affinity towards several neurotransmitter systems other than dopamine, may not add to the burden of negative symptoms, but they are not found to be primarily helpful in treating negative symptoms. Structural and functional imaging studies on negative symptoms have been largely inconsistent in their findings [3]. However, it is unlikely that findings from these studies would translate into development of therapeutic interventions in the foreseeable future. A host of pharmacological agents have been investigated for the treatment of negative symptoms [4]. Many have shown promise in small, largely preliminary studies. However, no agent has been shown to be consistently effective in sufficiently large studies. Cognitive behavior therapy (CBT) and social skills training (SST) have shown some promise in managing negative symptoms [5]. However, the optimism raised by these trials gets dampened when one considers the challenges involved in scaling up such therapies and making them accessible to a large number of patients that need such therapies [6]. Thus, it is understandable that an average clinician, who is faced with the challenge of negative symptoms in his/her patients, feels pessimistic about the prospects of helping them.

\section{Neurocognition, Social Cognition and Cognitive Organization}

Several meta-analyses have reported significant but modest association between negative symptoms and specific neurocognitive deficits [7]. Similarly, several studies have found significant association between social cognition and negative symptoms [8]. Cognitive remediation interventions do not typically involve negative symptoms as important outcome measure. However, cognitive remediation interventions have modest effect on negative symptoms too [9].

An alternative model of examining the cognitive deficits has been put forth recently [10]. According to this model, a number of non-neurocognitive factors influence the performance of individuals with schizophrenia on cognitive tests. These include motivation, effort, asociality, stress, negative emotions, and disorganized symptoms. The model posits that dysfunctional cognitive organization, i.e., dysfunctional attitudes, meanings and beliefs, underlie these moderating factors. There is evidence that recoveryoriented cognitive therapy (CT-R), designed to activate and strengthen the positive beliefs and inactivate and weaken the negative beliefs, results in improvement in neurocognitive test performance [11]. CT-R was also found to improve negative symptoms and global functioning and the gains were sustained after the therapy was withdrawn $[12,13]$. While this is promising, the issue of scalability remains relevant to this as well.

Thus, a number of non-neurocognitive factors influence the performance of individuals with schizophrenia on neurocognitive tests and on negative symptoms. What are the determinants of these factors? While the association of dysfunctional cognitive organization with poor neurocognitive performance and negative symptoms have been well-researched [10], there is dearth of research exploring the determinants of such beliefs and attitudes. It is possible that these may have biological underpinnings. There may be social determinants too- there is emerging evidence suggesting that shift in the sense of self and the world [14] and social exclusion [15] can contribute to poor performance of individuals with schizophrenia on neurocognitive tests. This line of thinking, though relatively new and needs more empirical support, is very compelling, given that persons with schizophrenia experience substantial disadvantage in the form of stigma, discrimination, exclusion, and social isolation [16], and the this can have profound impact on an individual's attitudes about one's own self worth and belief in him/herself. As a corollary, interventions targeting these have potential to improve cognitive as well as negative symptoms.

Secondary negative symptoms Introduction of the concept of primary and secondary negative symptoms at the end of the last century reduced the extent of pessimism regarding negative symptoms. The primary negative symptoms were considered as emerging from core neurobiological dysfunction of schizophrenia. The secondary negative symptoms were considered as having multiple sources - these include coping from the effects of positive symptoms, depression, anxiety, extrapyramidal adverse effects of antipsychotic medications and effects of environmental deprivation. Addressing these factors generally results in reduction of the burden of negative symptoms. 
Institutionalization and negative symptoms An important contributor to secondary negative symptoms is institutionalization [17]. Many factors associated with long-term institutionalization can potentially contribute to negative symptoms. These include loss of contact with the outside world, unstimulating environment, stereotyped routines with considerable periods of idleness, uniforms, paternalistic attitude of the staff, influence of labeling as 'mentally ill', fostering of self-image of being sick, invalidated, and incapacitated. These lead to gradual loss of individuality, drive, and motivation. Though the practice of institutionalizing persons with mental illness in mental health institutes has reduced over the past decades, in the absence of optimal community care, patients discharged from such institutes have been landing up alternative places, with not dissimilar environments.

"Institutionalization in the community"? Vast majority of persons with severe mental illness do not live in mental health institutes. In several countries, they have been discharged through the deinstitutionalization movement; in many other countries like India institutional care applied to a miniscule proportion of the population. However, factors, which contribute to the development of negative symptoms and personality change in the institutes, may operate in the community too, albeit with less intensity. Most patients with severe mental illnesses living in the community do not get optimal rehabilitation services. In the absence of this, many would face similar conditions as in mental health institutions. In most communities in the world, there is considerable degree of social stigma, discrimination, and denial of opportunity for persons with severe mental illnesses. There is widespread lack of reasonable accommodation in education and work spheres, leading to significant underachievement and unemployment. Social stigma and poor mental health literacy mean loss of opportunity to have friends and life partners. These create fertile grounds for these individuals to develop low self-esteem, self-stigma, depression, loss of drive and poor motivation. Not surprisingly, these add to the burden of secondary negative symptoms. As discussed above, many of these factors have been found to contribute to poor cognitive functions, which further affect community functioning.

Role of welfare and public engagement measures Several evidence-based psychosocial interventions have potential to improve community functioning and quality of life of persons with schizophrenia.
However, there are several barriers for a vast majority of persons with schizophrenia to avail these interventions [6]. In this context, the role of welfare and public engagement measures becomes critical. Effective community awareness and anti-stigma measures can contribute to healthier attitude of the public towards persons with mental illnesses. Active measures to improve inclusion of persons with disability due to mental illness in educational institutes and providing them employment opportunities with reasonable accommodation can alleviate exclusion and the consequent negative psychological impact. Provisions for their active inclusion in social, recreational, and healthpromoting activities including sports and cultural activities can go a long way in breaking the stereotypes about persons with mental illness and bring in change in their monotonous lives and thus pave way for opportunities to make friends and life partners. Finally, rights-based financial support in the form of disability pension and unemployment pension has potential to enhance their financial autonomy and help in leading dignified lives. These measures can turn out to be therapeutic against factors discussed in the previous paragraph that contribute to negative symptom burden. This, in turn, can improve functioning and quality of life of persons with schizophrenia.

Governments across the world have an obligation to implement these welfare and public engagement measures in the context of the UNCRPD. While these measures have been thought of as rights-based measures to ensure equal enjoyment of all human rights and fundamental freedoms by persons with disabilities, as a clinician-researcher, I can see the significant therapeutic role these measures may have for persons with severe mental illnesses. These should complement structured psychosocial interventions meant for these individuals, which, currently are available to only a miniscule proportion of them. If the governments comply with their obligation to implement such welfare and public engagement measures in the right spirit, one could hope for reduction in the contextual contributors towards negative symptoms of schizophrenia.

\section{Declarations}

Conflict of interest The author discloses no conflicts of interest. This work was not funded by any funding sources. 


\section{References}

1. Bobes J, Arango C, Garcia-Garcia M, Rejas J. Prevalence of negative symptoms in outpatients with schizophrenia spectrum disorders treated with antipsychotics in routine clinical practice: findings from the CLAMORS study. J Clin Psychiatry. 2009;71:280-6.

2. Kay SR, Fiszbein A, Opler LA. The positive and negative syndrome scale (PANSS) for schizophrenia. Schizophr Bull. 1987;13:261-76.

3. Galderisi S, Davidson M, Kahn RS, Mucci A, Boter H, Gheorghe MD, et al. Correlates of cognitive impairment in first episode schizophrenia: the EUFEST study. Schizophr Res. 2009;115:104-14.

4. Căpățină OO, Micluția IV, Fadgyas-stănculete M. Current perspectives in treating negative symptoms of schizophrenia: a narrative review. Exp Ther Med Spandidos Publ. 2021;21:1-1.

5. Elis O, Caponigro JM, Kring AM. Psychosocial treatments for negative symptoms in schizophrenia: current practices and future directions. Clin Psychol Rev . 2013;33:914-28.

6. Thirthalli J. Evidence-Based Psychosocial Intervention for Schizophrenia: Many a Barrier Between the Bench and the Bedside. J Psychosoc Rehabil Ment Health. 2016;3:27-30.

7. İnce $\mathrm{E}$, Üçok $\mathrm{A}$. Relationship between persistent negative symptoms and findings of neurocognition and neuroimaging in schizophrenia. Clin EEG Neurosci. 2018;49:27-35.

8. Pelletier-Baldelli A, Holt DJ. Are negative symptoms merely the "real world" consequences of deficits in social cognition? Schizophr Bull Oxford University Press US. 2020;46:23641.

9. Cella M, Preti A, Edwards C, Dow T, Wykes T. Cognitive remediation for negative symptoms of schizophrenia: a network meta-analysis. Clin Psychol Rev. 2017;52:43-51.

10. Beck AT, Himelstein R, Bredemeier K, Silverstein SM, Grant $\mathrm{P}$. What accounts for poor functioning in people with schizophrenia: a re-evaluation of the contributions of neurocognitive v. attitudinal and motivational factors. Psychol Med. 2018;48:2776-85.

11. Grant P, Perivoliotis D, Luther L, Bredemeier K, Beck A. Rapid improvement in beliefs, mood, and performance following an experimental success experience in an analogue test of recovery-oriented cognitive therapy. Psychol Med. 2018;48:261.

12. Grant PM, Huh GA, Perivoliotis D, Stolar NM, Beck AT. Randomized trial to evaluate the efficacy of cognitive therapy for low-functioning patients with schizophrenia. Arch Gen Psychiatr Am Med Assoc. 2012;69:121-7.

13. Grant PM, Bredemeier K, Beck AT. Six-month follow-up of recovery-oriented cognitive therapy for low-functioning individuals with schizophrenia. Psychiatr Serv Am Psychiatric Assoc. 2017;68:997-1002.

14. Juckel G, Sass L, Heinz A. Anhedonia, self-experience in schizophrenia, and implications for treatment. Pharmacopsychiatry. 2003;36:176-80.

15. Reddy F, Reavis E, Polon N, Morales J, Green M. 102. The Cognitive Costs of Social Exclusion in Schizophrenia. Schizophr Bull. 2017;43:S54.

16. Thirthalli J, Kumar CN. Stigma and disability in schizophrenia: developing countries' perspective. Int Rev Psychiatry Abingdon Engl England. 2012;24:423-40.

17. Oshima I, Mino Y, Inomata Y. Institutionalisation and schizophrenia in Japan: social environments and negative symptoms: nationwide survey of in-patients. Br J Psychiatry . 2003;183:50-6.

Publisher's Note Springer Nature remains neutral with regard to jurisdictional claims in published maps and institutional affiliations. 